\title{
Increased CD11b and Decreased CD62L in Blood and Airway Neutrophils from Long-Term Smokers with and without COPD
}

\author{
Marit Stockfelt ${ }^{a, b}$ Karin Christenson ${ }^{c}$ Anders Andersson $^{d, e}$ Lena Björkman $^{b}$ \\ Médea Padra ${ }^{f}$ Bettina Brundin ${ }^{g}$ Koustav Ganguly ${ }^{g}, \mathrm{~h}$ Helga Asgeirsdottir ${ }^{\mathrm{a}}$ \\ Sara Lindén ${ }^{f}$ Ingemar Qvarfordt ${ }^{\mathrm{a}}$ Johan Bylund ${ }^{c}$ Anders Lindén ${ }^{9, i}$ \\ aSection of Respiratory Medicine and Allergology, Department of Internal Medicine and Clinical Nutrition, \\ Institute of Medicine, Sahlgrenska Academy at the University of Gothenburg, Gothenburg, Sweden; ${ }^{\text {bDepartment }}$ \\ of Rheumatology and Inflammation Research, Institute of Medicine, Sahlgrenska Academy at the University of \\ Gothenburg, Gothenburg, Sweden; 'Department of Oral Microbiology \& Immunology, Institute of Odontology, \\ Sahlgrenska Academy at the University of Gothenburg, Gothenburg, Sweden; ${ }^{d}$ COPD Center, Department of

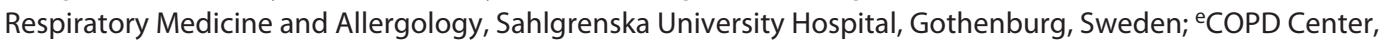 \\ Department of Internal Medicine and Clinical Nutrition, Institute of Medicine, Sahlgrenska Academy at the

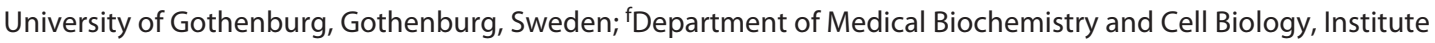 \\ of Biomedicine, Sahlgrenska Academy at the University of Gothenburg, Gothenburg, Sweden; 9Unit for Lung and \\ Airway Research, Institute of Environmental Medicine, Karolinska Institutet, Stockholm, Sweden; ' Unit of Integrative \\ Toxicology, Institute of Environmental Medicine (IMM), Karolinska Institutet, Stockholm, Sweden; 'Department of \\ Respiratory Medicine and Allergy, Karolinska University Hospital, Stockholm, Sweden
}

\section{Keywords}

Chronic obstructive pulmonary disease $\cdot$ Bronchoalveolar lavage $\cdot$ Granulocyte $\cdot$ Priming $\cdot$ CD11b $\cdot$ CD62L

\footnotetext{
Abstract

There is incomplete mechanistic understanding of the mobilization of neutrophils in the systemic and local compartment in smokers with chronic obstructive pulmonary disease (COPD). In this pilot study, we characterized how the adhesion molecules CD11b and CD62L, surface markers indicative of priming, are altered as neutrophils extravasate, and whether surface density of CD11b and CD62L differs between long-term tobacco smokers (LTS) with and without COPD compared with healthy never-smokers (HNS). Unstimulated blood neutrophils from LTS with $(n=5)$ and without $(n=9)$ COPD displayed lower surface density of CD62L compared with HNS $(n=8)$. In addition, surface density of CD11b
}

was higher in bronchoalveolar lavage (BAL) neutrophils from LTS without COPD compared with those with COPD and HNS. Moreover, in BAL neutrophils from all study groups, CD62L was lower compared with matched blood neutrophils. In addition, BAL neutrophils responded with a further decrease in CD62L to ex vivo TNF stimulation. Thus, neutrophils in the airway lumen display a higher state of priming than systemic neutrophils and bear the potential to be further primed by local cytokines even with no smoking or the presence of COPD, findings that may represent a universal host defense mechanism against local bacteria. Moreover, systemic neutrophils are primed in LTS regardless of COPD. Further studies in larger materials are warranted to determine whether the priming of neutrophils is protective against COPD or merely preceding it.

C 2020 The Author(s)

Published by S. Karger AG, Basel
(C) 2020 The Author(s)

Published by S. Karger AG, Basel

This is an Open Access article licensed under the Creative Commons Attribution-NonCommercial-4.0 International License (CC BY-NC) (http://www.karger.com/Services/OpenAccessLicense), applicable to the online version of the article only. Usage and distribution for commercial purposes requires written permission.
Marit Stockfelt

Department of Rheumatology, Institute of Medicine Sahlgrenska Academy at the University of Gothenburg Guldhedsgatan 10A, SE-405 30 Göteborg (Sweden) marit.stockfelt@gu.se 


\section{Background}

In the Western world, long-term tobacco smoking is the dominating cause of chronic obstructive pulmonary disease (COPD) [1]. This debilitating disease is characterized by irreversible airflow obstruction that often progresses over time, and COPD is predicted to soon become the third leading cause of disease-related mortality worldwide $[1,2]$. Apart from smoking cessation, there are still no truly effective therapeutic strategies against COPD.

Long-term tobacco smoking causes chronic inflammation in the airways, and, in general, this inflammation emerges as more pronounced in tobacco smokers with COPD than in those without it [3]. Multiple host defense mechanisms, including airway macrophages [4], neutrophils [5], and antibacterial secretion of the airway epithelium [6], are suppressed by tobacco smoke. Long-term tobacco smokers (LTS) with COPD display an accumulation of neutrophils in the systemic (blood) [7] as well as local (bronchoalveolar) compartments, and the increased local neutrophil number is inversely correlated with FEV1 [8]. Given this, as well as the pathogenically relevant effects that neutrophils can account for, these innate effector cells have been attributed a causative role in COPD $[9,10]$. In line with such a role, the local accumulation of neutrophils is further increased during acute exacerbations of COPD [11], but it is still unclear to what extent this is due to the immunological aberration in COPD per se, the presumed infection triggering the exacerbation, or both [12].

During their extravasation from the systemic to the local compartment, neutrophils are typically primed, resulting in a preactivated and hyperresponsive state [13]. This type of priming is in part mediated through the mobilization of intracellular granules which leads to a reorganization of surface markers, and by this mechanism, the integrin $\mathrm{CD} 11 \mathrm{~b}$ is upregulated through release of granules and secretory vesicles [14], while the cell adhesion molecule CD62L (L-selectin) is cleaved off by proteolytic shedding [15]. Thus, enhanced surface density of $\mathrm{CD} 11 \mathrm{~b}$ and lower levels of CD62L are a pattern typical for primed neutrophils. Notably, a similar type of altered surface density of CD11b and CD62L can be accomplished by ex vivo stimulation of resting blood neutrophils with the proinflammatory cytokine TNF [16]. However, at present, it is unclear to what extent the priming of neutrophils differs for LTS with and without COPD.

Given that there is still incomplete understanding of the immunological mechanisms associated with the mobilization and priming of neutrophils in the systemic (blood) as well as local (bronchoalveolar) compartments in smokers with COPD, we aimed to further characterize these phenomena. Specifically, we performed the current pilot study to characterize surface markers indicative of priming in neutrophils matched from blood and bronchoalveolar lavage (BAL) samples within each study subject belonging either to a group of LTS, with or without COPD, or healthy never-smokers (HNS).

\section{Methods}

\section{Study Population and Design}

The current study was conducted in accordance with the Declaration of Helsinki, and the study protocol was approved by the Regional Committee for Ethical Review in Gothenburg, Sweden (Diary No. 968-11). All subjects provided their oral and written informed consent before being included and examined.

A detailed description of the study protocol has been published elsewhere [17]. At the individual level, certain data have been utilized in the referred publication but within the scope of another scientific aim. In brief, 24 subjects were recruited for the current pilot study by advertising in the daily press. Two subjects were excluded: one was due to inability to undergo the bronchoscopy and the other had chronic bronchitis and could therefore not be included in the group of LTS without significant lung disease. The final population sample for this pilot study consisted of 8 HNS, 9 LTS without COPD, and 5 LTS with COPD (Table 1). The included LTS with and without COPD were all long-term and current tobacco smokers with a tobacco load of $\geq 20$ pack-years and at least 5 cigarettes per day during the last 5 years. The smokers were allocated to the LTS without COPD group if they displayed a $\mathrm{FEV}_{1} /$ FVC (FEV\%) ratio of $\geq 70 \%$ after bronchodilation and fulfilled GOLD criteria for COPD including typical respiratory symptoms [2]. The included subjects from the groups of LTS without COPD and the group of HNS did not have respiratory symptoms. No included subject had clinical symptoms of respiratory allergy.

All study subjects were investigated during a stable clinical state. A negative history of atopy as well as a negative screening test for specific IgE antibodies against inhaled allergens (Phadiatop ${ }^{\mathrm{TM}}$, Phadia, Uppsala, Sweden) was required. Short-acting, inhaled bronchodilators constituted the only accepted medication, and thus, steroid therapy constituted an exclusion criterion. Accepted comorbidities included controlled depression, epilepsy, hypertension, hypothyroidism, osteoporosis, and past cancer ( $>5$ years ago). Exclusion criteria were pregnancy, use of wet snuff, or chewing tobacco as well as $>3$ respiratory infections during the last year prior to the current study. Each subject attended a first visit for medical examination, spirometry with bronchodilation, and chest X-ray and went through electrocardiography (ECG) before the bronchoscopy.

Bronchoscopy and Bronchoalveolar Lavage

Bronchoscopy was performed through the mouth with a flexible fiberoptic bronchoscope after local anesthesia with xylocaine and premedication with ketobemidone. The bronchoscope was wedged in a segmental bronchus (mid lobe or lingual), and BAL with 3 aliquots $(50 \mathrm{~mL}$ each, a total of $150 \mathrm{~mL}$ ) was performed. The BAL samples were retracted, collected, pooled, and kept on ice until further processing. 
Table 1. Clinical characteristics of the study population

\begin{tabular}{|c|c|c|c|}
\hline & HNS & LTS without COPD & LTS with COPD \\
\hline Subjects, $n$ & 8 & 9 & 5 \\
\hline Sex, female/male & $2 / 6$ & $6 / 3$ & $2 / 3$ \\
\hline Age, years & $49.5(44.8,62.3)$ & $66.5(60,69)$ & $59(55,64)$ \\
\hline $\mathrm{BMI}, \mathrm{kg} / \mathrm{m}^{2}$ & $24.5(22.8,27)$ & $24(23.1,25.2)$ & $26(21,28)$ \\
\hline Historic tobacco load, pack-years & $0(0)$ & $37.3(25,40)^{1}$ & $35(30,40)^{1}$ \\
\hline $\mathrm{FEV}_{1} \%$ predicted & $110(105,115)$ & $100(97,112)$ & $78(77,85)^{3}$ \\
\hline FEV1/FVC, FEV\% & $79(73.5,82.3)$ & $74.5(72,77)$ & $61(57,61)^{2,4}$ \\
\hline $\mathrm{CO}$ diffusion capacity, $\mathrm{mmol} \min ^{-1} \mathrm{kPa}^{-1}$ & $9.4(6.7,10)$ & $6(5.7,6.6)$ & $7.6(6.7,8.2)$ \\
\hline $\mathrm{CO}$ diffusion capacity/VA & $1.3(1.2,1.4)$ & $1.2(1.1,1.3)$ & $1.2(1,1.3)$ \\
\hline
\end{tabular}

Data are presented as median (quartile 1, quartile 3) with statistical evaluation using the Kruskall-Wallis test with Dunn's multiple comparison test. HNS, healthy never-smokers; LTS, long-term smokers; COPD, chronic obstructive pulmonary disease. ${ }^{1} p<0.001$ compared with HNS. ${ }^{2} p<0.01$ compared with HNS. ${ }^{3} p<0.05$ compared with HNS. ${ }^{4} p<0.05$ compared with LTS.

Fig. 1. Illustration of the gating strategy. Blood neutrophils (a) were gated based on FSC/SSC characteristics. The same gate was used for BAL neutrophils (c). Autofluorescent (in the FL4 detector) BAL cells (d) were removed from the analyses, using the gate from blood neutrophils which are not autofluorescent (b). BAL, bronchoalveolar lavage; FSC/SSC, forward scatter/side scatter.

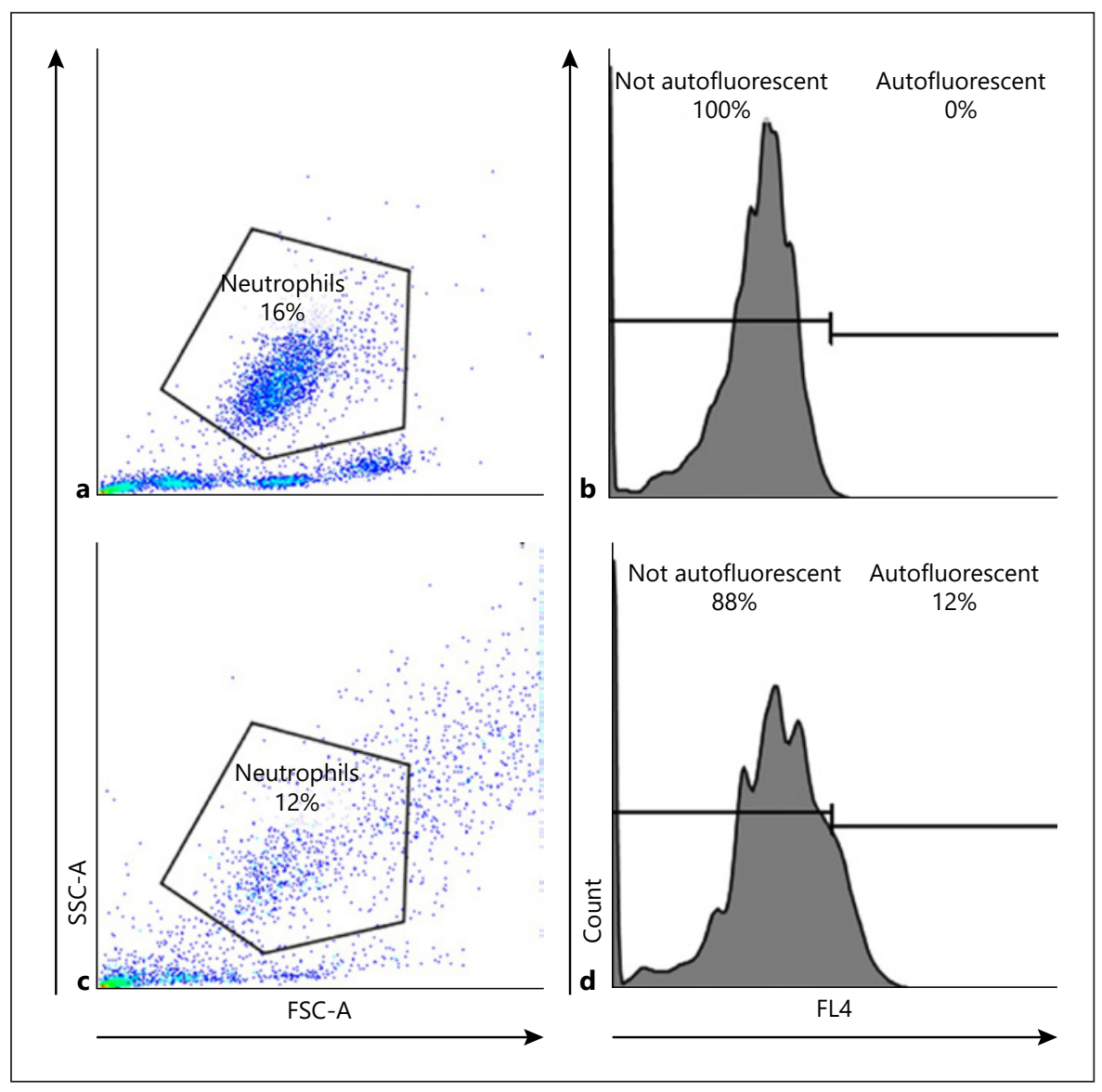

Handling of Blood and BAL Samples

Peripheral blood was sampled in ethylenediaminetetraacetic acid (EDTA) vacutainer tubes immediately before the bronchoscopy. For the determination of the concentrations of leukocyte subsets, differential cell counts in a flow cytometer were used (Ad- via 2120i, Kemlab, Sahlgrenska University Hospital, Gothenburg, Sweden). One BAL aliquot was cytocentrifuged and stained with May-Grünwald-Giemsa for a differential cell count of 200 cells at the cytology department, Sahlgrenska University Hospital, and the total number of BAL cells was quantified in a Bürker chamber. 
Table 2. Cellular distribution in blood samples

\begin{tabular}{llllll}
\hline & HNS & $\begin{array}{l}\text { LTS without } \\
\text { COPD }\end{array}$ & $\begin{array}{l}p \text { value } \\
\text { versus HNS }\end{array}$ & $\begin{array}{l}\text { LTS with } \\
\text { COPD }\end{array}$ & $\begin{array}{l}p \text { value } \\
\text { versus HNS }\end{array}$ \\
\hline Neutrophils, $10^{9} / \mathrm{L}$ & $2.9(2.6,3.2)$ & $3.5(3.2,3.8)$ & 0.047 & $4.3(3.4,4.3)$ & 0.037 \\
Lymphocytes, $10^{9} / \mathrm{L}$ & $1.8(1.3,2.1)$ & $1.9(1.6,2.5)$ & 0.68 & $2.6(2.4,3.1)$ & 0.048 \\
Monocytes, $10^{9} / \mathrm{L}$ & $0.4(0.3-0.4)$ & $0.4(0.4,0.5)$ & 0.55 & $0.5(0.4,0.6)$ & 0.25 \\
Eosinophils, $10^{9} / \mathrm{L}$ & $0.2(0.2-0.2)$ & $0.2(0.1,0.3)$ & $>0.99$ & $0.3(0.3,0.4)$ & 0.38 \\
Basophils, $10^{9} / \mathrm{L}$ & $0(0,0)$ & $0(0,0)$ & 0.49 & $0(0,0.1)$ & 0.15 \\
\hline
\end{tabular}

Concentrations of leukocyte subsets $\left(\times 10^{9} / \mathrm{L}\right)$ in blood samples from HNS and LTS with and without COPD. Data are presented as median (quartile 1, quartile 3) with statistical evaluation using the Kruskall-Wallis test with Dunn's multiple comparison test. HNS, healthy never-smokers; LTS, long-term smokers; COPD, chronic obstructive pulmonary disease.

\section{U-PLEX ${ }^{\circledR}$ Analysis of TNF Protein}

Four milliliters $(\mathrm{mL})$ of cell-free BAL fluid from each subject was concentrated $(\times 6.7-10.5)$ using the Amicon ${ }^{\circledR}$ Ultra-4 Centrifugal Filter Device (UFC801024; Merck, Darmstadt, Germany) according to the manufacturer's instructions, and cytokine concentrations in the BAL fluid were corrected for this concentration factor. TNF was quantified in the concentrated and cell-free BAL fluid or plasma samples from peripheral blood, using the U-PLEX assay $^{\circledR}$, Meso Scale Discovery ${ }^{\mathrm{TM}}$ platform (Cat No. K15067-L1; Meso Scale Diagnostics, Rockville, MD, USA) according to the manufacturer's instructions.

\section{Ficoll Separation and Cell Stimulation}

The BAL cell pellet was suspended in 7.5-mL PBS. Equal volume of Ficoll ${ }^{\circledR}$-Paque PLUS (GE Healthcare, Chicago, IL, USA) was layered under the BAL with a syringe without mixing and was thereafter centrifuged $\left(931 \mathrm{~g}, 15 \mathrm{~min}\right.$ at $\left.4^{\circ} \mathrm{C}\right)$. The upper layer and the Ficoll layer were removed with a sterile Pasteur pipette, and the remaining cell pellet was suspended in $30-\mathrm{mL}$ PBS. Following another centrifugation step $\left(335 \mathrm{~g}, 10 \mathrm{~min}\right.$ at $\left.4^{\circ} \mathrm{C}\right)$, the supernatant was discarded and the layer with neutrophils (polymorphonuclear leukocytes) was suspended in Krebs-Ringer glucose buffer (KRG) supplemented with $\mathrm{Ca}^{2+}$. These BAL neutrophils as well as the whole blood cells were incubated at $37^{\circ} \mathrm{C}$ with an addition of 10 ng/mL TNF (Cat No. T6674; Sigma, Saint Louis, MO, USA) or left unstimulated in room temperature $(20 \mathrm{~min})$. Red blood cells were lysed using BD FACS ${ }^{\mathrm{TM}}$ Lysing Solution (Cat No. 349202; BD Biosciences, Franklin Lakes, NJ, USA) (diluted 1:10 in distilled water) for 15 min, once for BAL cells and twice for whole blood cells. The cells were then resuspended in PBS before analysis with flow cytometry.

\section{Flow Cytometry}

Whole blood cells and BAL neutrophils were stained with fluorochrome-conjugated antibodies, anti-CD11b PE (Cat No. 333142) or anti-CD62L PE (Cat No. 341012) (both from BD Biosciences, Franklin Lakes, NJ, USA). Samples were resuspended in 250- $\mu \mathrm{L}$ PBS with EDTA and analyzed using a BD Accuri ${ }^{\mathrm{TM}}$ C6 (BD Biosciences, Franklin Lakes, NJ, USA) flow cytometer and FlowJo software (Tree Star, Ashland, OR, USA). Neutrophils from blood were gated based on forward scatter/side scatter (FSC/SSC) characteristics. For BAL cells, the neutrophil gate from blood cells was used in combination with exclusion of autofluorescent cells since alveolar macrophages, but not neutrophils, are highly autofluorescent. The principal gating strategy is shown in Figure 1. The surface density of CD11b and CD62L is presented as mean fluorescence intensity (MFI). BAL neutrophils from the first-recruited HNS subject were not stimulated by TNF ex vivo due to human error and removed from the analysis.

\section{Statistical Analysis}

Descriptive statistics on subject characteristics, lung function data, and differential counts were presented as median (quartile 1, quartile 3), and for analytical statistics regarding differential counts, the Kruskal-Wallis test was used with Dunn's multiple comparison tests. Data were analyzed using GraphPad prism 6 (GraphPad Software Inc., San Diego, CA, USA).

For analytical statistics regarding neutrophil surface markers and TNF concentration, two-way variance analysis was used, where the 2 factors were group and stimulated (yes/no), respectively. An interaction term between the factors was added to the model. The SAS procedure MIXED was used for the calculations (SAS version 9.4; SAS Institute Inc., Gary, NC, USA). The letter $n$ refers to the number of independent observations and equals the number of subjects. A $p$ value of $<0.05$ was regarded as statistically significant.

\section{Results}

\section{Characteristics of the Study Population}

The clinical characteristics of the study population are shown in Table 1 . The recruited subjects had an age range of 41-74 years. The groups of LTS with and without COPD had a very similar historic tobacco load. The included patients in the group of LTS with COPD corresponded to GOLD stages 1 and 2 [2].

\section{Cellular Distribution and TNF Concentration in Blood and BAL Samples}

The concentration of blood neutrophils was modestly increased in the group of LTS, both with and without 
Table 3. Cellular distribution in BAL samples

\begin{tabular}{lccccl}
\hline & HNS & $\begin{array}{l}\text { LTS without } \\
\text { COPD }\end{array}$ & $\begin{array}{l}p \text { value } \\
\text { versus HNS }\end{array}$ & $\begin{array}{l}\text { LTS with } \\
\text { COPD }\end{array}$ & $\begin{array}{l}p \text { value } \\
\text { versus HNS }\end{array}$ \\
\hline Neutrophils, $\times 10^{4} / \mathrm{mL}$ & $0.3(0.2,0.6)$ & $0.3(0.3,0.5)$ & $>0.99$ & $2.2(0.9,3.9)$ & 0.02 \\
Lymphocytes, $\times 10^{4} / \mathrm{mL}$ & $3.1(0.3,6.4)$ & $0.2(0,0.5)$ & 0.044 & $0.4(0.2,0.7)$ & 0.53 \\
Macrophages, $\times 10^{4} / \mathrm{mL}$ & $11.5(9.1,14.5)$ & $21.0(20.6,33.3)$ & 0.0025 & $21.3(17.5,25)$ & 0.027 \\
Eosinophils, $\times 10^{4} / \mathrm{mL}$ & $0(0,0.1)$ & $0(0,0)$ & 0.48 & $0(0-1.7)$ & 0.71 \\
\hline
\end{tabular}

Concentrations of leukocyte subsets $\left(\times 10^{4} / \mathrm{mL}\right)$ in BAL samples from HNS and LTS with and without COPD. Data are presented as median (quartile 1, quartile 3) with statistical evaluation using the Kruskall-Wallis test with Dunn's multiple comparison test. BAL, bronchoalveolar lavage; HNS, healthy never-smokers; LTS, long-term smokers; COPD, chronic obstructive pulmonary disease.

Table 4. Concentration of TNF protein $(\mathrm{pg} / \mathrm{mL})$ in plasma and cell-free BAL samples from HNS and LTS with and without COPD

\begin{tabular}{|c|c|c|c|c|c|}
\hline & HNS & $\begin{array}{l}\text { LTS without } \\
\text { COPD }\end{array}$ & $\begin{array}{l}p \text { value } \\
\text { versus HNS }\end{array}$ & $\begin{array}{l}\text { LTS with } \\
\text { COPD }\end{array}$ & $\begin{array}{l}p \text { value } \\
\text { versus HNS }\end{array}$ \\
\hline Plasma & $2.6(2.4,3.4)$ & $3.4(2.9,4.2)$ & 0.4556 & $2.5(1.9,2.5)$ & 0.6752 \\
\hline BAL & $0.2(0.1,0.5)$ & $0.2(0.1,0.2)$ & $>0.9999$ & $0.3(0.2,0.6)$ & $>0.9999$ \\
\hline
\end{tabular}

Data are presented as median (quartile 1, quartile 3) with statistical evaluation using the Kruskall-Wallis test with Dunn's multiple comparison test. BAL, bronchoalveolar lavage; HNS, healthy never-smokers; LTS, longterm smokers; COPD, chronic obstructive pulmonary disease.

COPD, compared with the control group of HNS. The concentration of blood lymphocytes was modestly increased in LTS with COPD compared with HNS. Regarding eosinophils, all subjects were within the expected limits of the method except for 1 patient with COPD who displayed an eosinophil value of 0.6 , and there were no statistical differences between the groups (Table 2). The concentration of BAL neutrophils was 7-fold higher in the group of LTS with COPD compared with HNS, while lymphocytes were increased in HNS in comparison with the other groups and macrophages were almost doubled in LTS with and without COPD compared with HNS (Table 3). The level of TNF was measurable in blood and BAL fluid in all groups with no statistically significant grouprelated differences (Table 4).

\section{Surface Markers Indicative of Priming on Blood \\ Neutrophils}

The density of the surface markers CD11b and CD62L on blood neutrophils was assessed utilizing flow cytometry after gating of blood neutrophils according to FSC/ SSC. The groups of LTS with and without COPD dis- played clearly lower surface density of CD62L on unstimulated blood neutrophils compared with the control group of HNS (Fig. 2b). Compared with TNF-stimulated blood neutrophils, unstimulated blood neutrophils displayed lower CD11b surface density (Fig. 2a) and higher CD62L surface density (Fig. 2b), and short stimulation ex vivo with TNF upregulated $\mathrm{CD} 11 \mathrm{~b}$ and downregulated CD62L in blood neutrophils in all groups. These ex vivo effects of TNF displayed no clear group-related differences $(p>0.05)$.

\section{Surface Markers Indicative of Priming on $B A L$ \\ Neutrophils}

Compared with matched blood neutrophils, unstimulated BAL neutrophils displayed higher surface density of CD11b in both the group of LTS without COPD and the group of HNS, with a similar order of magnitude observed in LTS with COPD ( $p=0.04$ for HNS; $p<0.0001$ for LTS without COPD; $p=0.16$ for LTS with COPD) (Fig. 3a). Compared with matched blood neutrophils, unstimulated BAL neutrophils displayed lower expression of CD62L ( $p<0.0001$ for all groups) (Fig. 3b). 


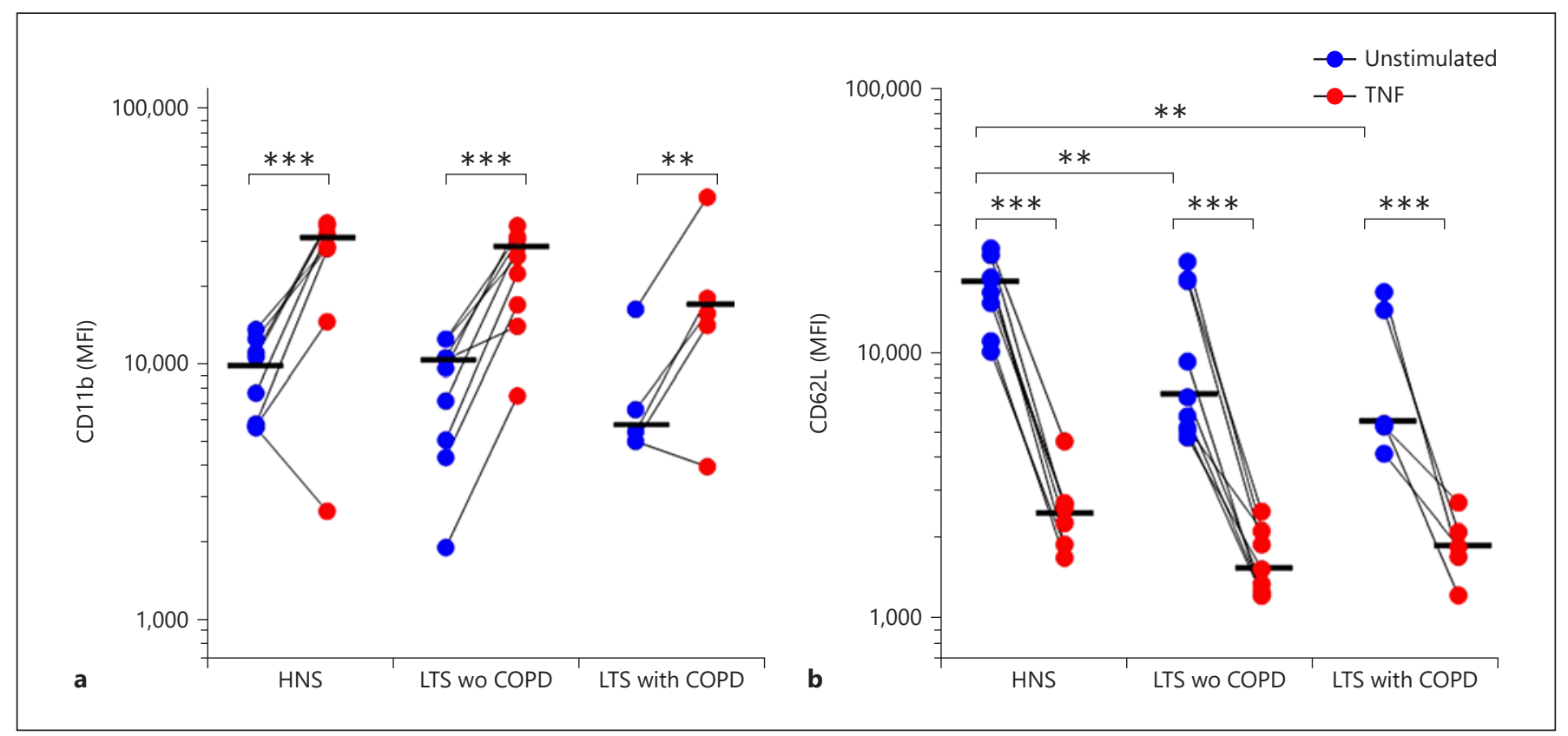

Fig. 2. Surface markers indicative of priming on blood neutrophils. Surface density (MFI) of CD11b (a) and CD62L (b) on blood neutrophils from HNS and LTS with and without COPD. The cells were either unstimulated (in blue) or stimulated (in red) with TNF at $37^{\circ} \mathrm{C}$, stained with antibodies against CD11b or CD62L and an-

Unstimulated BAL neutrophils from the group of LTS without COPD displayed significantly higher surface density of CD11b compared with that observed in the group of LTS with COPD and the control group of HNS, respectively (Fig. 4a). The BAL neutrophils from the group of LTS without COPD also displayed higher surface density of CD62L compared with that observed in the group of LTS with COPD but not HNS (Fig. 4b). Finally, stimulation with TNF ex vivo further increased surface density of CD11b in the group of LTS with COPD (Fig. 4a) and further decreased surface density of CD62L in all study groups (Fig. 4b).

\section{Discussion}

Blood neutrophils from LTS with and without COPD displayed a higher state of priming compared with cells from HNS, as indicated by a lower surface density for $\mathrm{CD} 62 \mathrm{~L}$, which is cleaved from the cell surface during priming. This lower surface density of CD62L indicates a slight priming of neutrophils in the systemic compartment, presumably reflective of other aspects of the sys- alyzed by flow cytometry based on the neutrophil gate. Data are presented as individual values with median, ${ }^{*} p<0.05,{ }^{* *} p<0.01$, and ${ }^{* * *} p<0.001$, two-way variance analysis. MFI, mean fluorescence intensity; HNS, healthy never-smokers; LTS, long-term smokers; COPD, chronic obstructive pulmonary disease.

temic inflammation observed in LTS with and without COPD [18]. From a hypothetical point of view, this priming means a higher sensitivity to activation and may facilitate the extravasation of systemic blood neutrophils into the local compartment of the airways. Moreover, stimulation with TNF ex vivo led to higher surface density of CD11b and lower surface density of CD62L in blood neutrophils from all study groups, as expected [16]. Just like our current study, a previous study on patients with COPD indicated enhanced priming in the form of lower surface density of CD62L in blood neutrophils from these patients, without the accompanying increase in CD11b [19]. However, in that study, there was no comparative group with LTS without COPD. Furthermore, another previous study indicated increased priming in the form of higher CD11b in blood neutrophils from patients with COPD in comparison with healthy controls [20].

In all groups of our current study, the surface density of CD62L was lower on neutrophils in the airways than on matched blood neutrophils, indicating priming caused by extravasation from the systemic to the local compartment. This fits well with the standard model whereby CD62L is in fact cleaved off before extravasation over the endothe- 


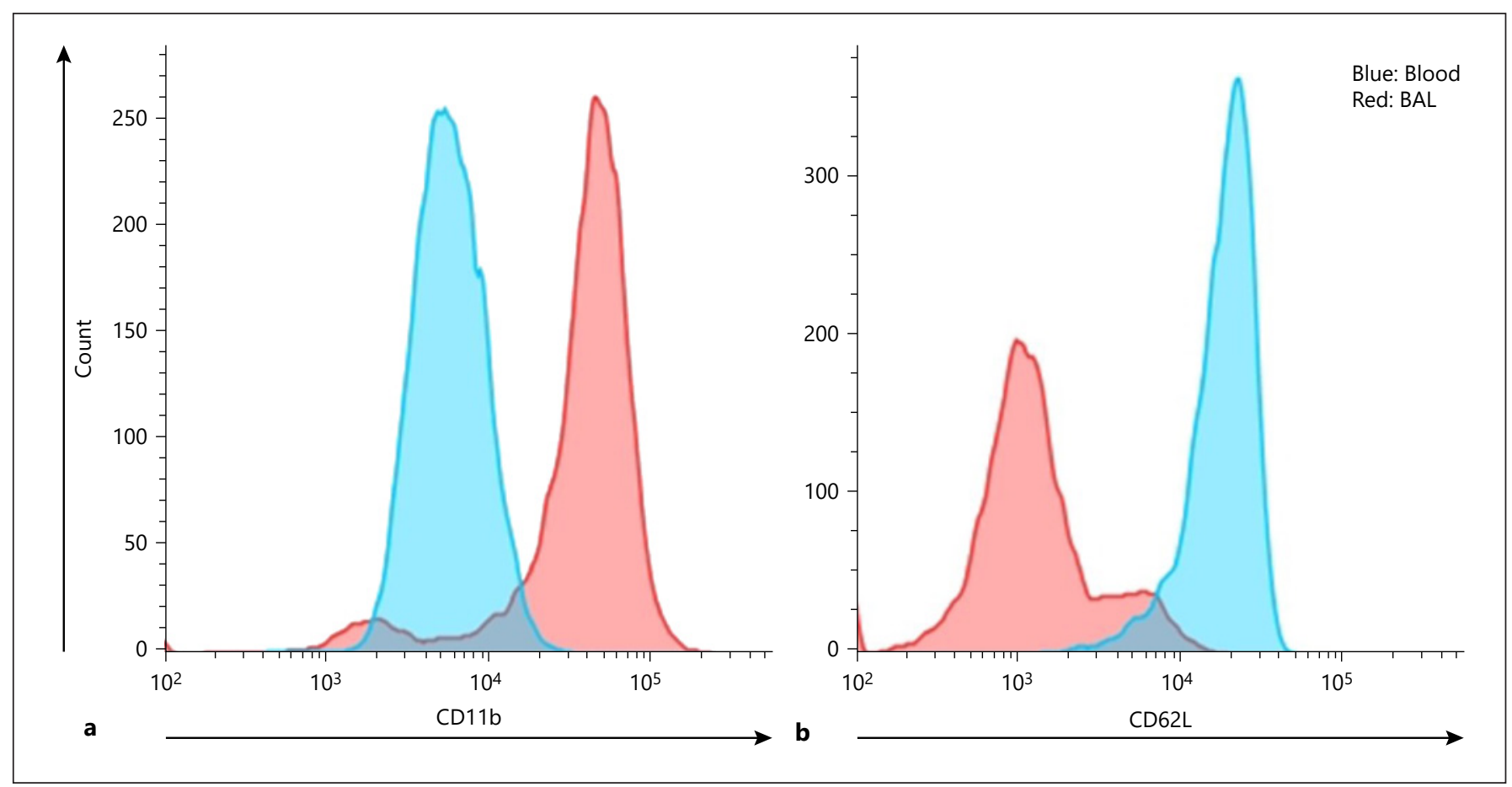

Fig. 3. Surface markers indicative of priming on blood and BAL neutrophils. The BAL neutrophils (in red) displayed higher surface density of CD11b (a) and lower surface density of CD62L (b) compared with blood neutrophils(in blue) of the same individual donor, in all groups. These graphs show representative flow cytometry histograms from a HNS. BAL, bronchoalveolar lavage; HNS, healthy never-smoker.

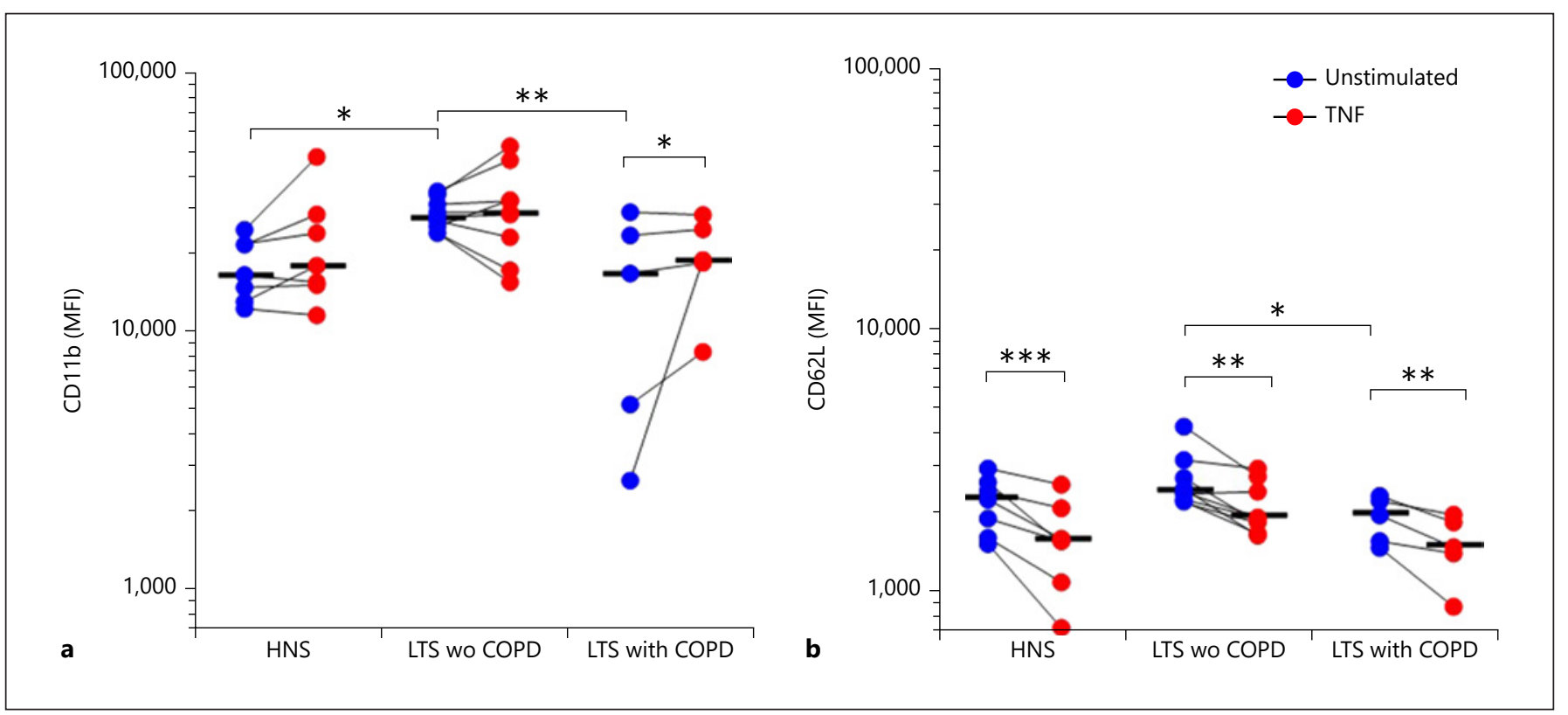

Fig. 4. Surface markers indicative of priming on BAL neutrophils. Surface density of CD11b (a) and CD62L (b) on BAL neutrophils. The cells were separated from BAL samples using Ficoll and unstimulated (in blue) or stimulated (in red) with TNF at $37^{\circ} \mathrm{C}$, stained with antibodies against CD11b or CD62L and analyzed by flow cytometry based on the neutrophil gate. Data are presented as individual values with median, ${ }^{*} p<0.05,{ }^{* *} p<0.01$, and $* * * p<0.001$, two-way variance analysis. BAL, bronchoalveolar lavage. 
lial layer and that the soluble form of the molecule can be found in circulation rather than in tissue exudates [15]. We found detectable levels of TNF in all groups, with no statistically significant group-related differences. The samples were collected during a stable phase of COPD disease meaning that fewer patients would be expected to have bacterial growth. During an infection, levels of TNF would be expected to rise. Here, the short-term stimulation with TNF in vitro was utilized as a tool to achieve full priming of neutrophils in terms of maximally increased surface CD11b and complete lack of CD62L. This is also what we observed in our samples of blood neutrophils, which reassures us that our experimental model was responding in accordance to what is previously known from the literature. Interestingly, stimulation with TNF ex vivo led to even lower surface density of CD62L in airway neutrophils from all groups. Clearly, this observation indicates that airway neutrophils are not completely primed by the extravasation process but can be further affected by the local inflammatory milieu. In this manner, the phenotype of airway neutrophils is not unlike that of neutrophils from synovial fluid of patients with arthritis, neutrophils that can be further primed by TNF as well [21]. In contrast, local neutrophils in skin models are completely primed and do not respond to local stimulation in the same way [16]. We suspect that the demonstrated sensitivity to local stimulation of airway neutrophils is indicative of an important mechanism in local host defense against bacteria in the airways, a possibility that deserves further evaluation in translational studies.

We also found that the surface density of the activation marker CD11b, which becomes higher during priming through degranulation, became indeed higher in blood neutrophils after stimulation with TNF, thus mirroring the lower surface density of CD62L. It is noteworthy that we found the surface density of CD11b to be higher in airway neutrophils from LTS without COPD compared with HNS and LTS with COPD. This does indicate that there is increased priming in local airway neutrophils from LTS without COPD only. Given that the historic exposure to tobacco smoke is a critical confounder that was similar in the LTS with and without COPD in our study, this observation forwards several feasible interpretations. One interpretation is that this type of increased priming is protective against the development of COPD. However, the surface density of CD62L in airway neutrophils of smokers with COPD was not lower here; this surface density actually displayed a somewhat higher level, and the difference was statistically significant for LTS without COPD compared with LTS with COPD. Typically, the higher

Neutrophil Priming in Smokers
CD11b is expected to be accompanied by a lower CD62L but our current observation lends support to the idea that although these processes often occur simultaneously, the two events are not strictly linked in mechanistic terms. Along these lines, the surface density of CD11b becomes higher through degranulation [14], whereas the surface density of CD62L becomes lower when it is cleaved through proteolytic shedding [15]. Interestingly, there is evidence that the regulation of priming molecules is stimuli dependent in peripheral neutrophils, where low doses of platelet-activating factor exert an impact on CD11b but not CD62L. In contrast, low doses of endotoxin exert an impact on CD62L but not CD11b [22]. Taken together, these observations support a separate regulation of integrins and selectins in neutrophils.

The current pilot study has certain limitations. One limitation is the relatively small sample size of the cohort, comprising 8 HNS, 9 LTS without COPD, and 5 LTS with COPD. Although these study groups were similar with respect to BMI, they differed slightly in that the LTS without COPD tended to be older and had a larger proportion of female participants. To the best of our knowledge, there are no published studies of gender differences in the surface density of CD11b and CD62L, and the current study material is too small to elucidate the role of gender. Another limitation is that although the flow cytometry gating was used to identify airway neutrophils, we cannot completely exclude the possibility that occasional, small, non-autofluorescent monocytes/macrophages were included among the neutrophils, thereby limiting the purity of the cells that were studied ex vivo. In contrast, we think that it was a clear advantage of the study that it included patients with mild-to-moderate COPD only. A main reason to study patients with milder COPD is to gain an insight into early pathogenic alterations of importance for the disease initiation and early development, alterations that may be reversible. These events are likely to occur prior to definitive remodeling and degradation of tissue due to severe and sustained inflammation that may lead to an irreversible end stage of disease. However, it can still be argued that the downside of our current approach is that we may miss developments that are evident only even later in the course of the disease.

In conclusion, the results of this pilot study indicate that systemic blood neutrophils in LTS with and without COPD have a higher state of priming compared with those in HNS, a finding that adds yet another sign of systemic inflammation to those previously reported in LTS with and without COPD. Moreover, the current results suggest that the higher state of priming in systemic blood 
neutrophils is partly mirrored in local airway neutrophils from LTS, a novel finding suggestive of increased sensitivity to activation for local airway neutrophils in LTS. Further studies will be required to determine whether this type of increased neutrophil priming represents a protective factor against the pathogenic mechanisms behind COPD, or merely an immunological phenomenon that precedes the clinical manifestation of this disease. Finally, for all three study groups, local airway neutrophils may be more primed than blood neutrophils are, but they can still be further primed ex vivo by TNF. This suggests that mere extravasation from the systemic blood to the local airway compartment is not enough to fully prime neutrophils. We propose that this potential of airway neutrophils to be further primed by local cytokines represents an important host defense mechanism against local bacteria, a possibility that warrants further evaluation in future studies.

\section{Acknowledgements}

The authors gratefully acknowledge Monika Crona and Dorota Persson for their assistance with the study.

\section{Statement of Ethics}

This study was conducted ethically in accordance with the World Medical Association Declaration of Helsinki, and the study protocol was approved by the Regional Committee for Ethical Review in Gothenburg, Sweden (\# 968-11). All subjects provided their written and oral informed consent.

\section{Disclosure Statement}

The authors have no conflicts of interest to declare.

\section{Funding Sources}

This work obtained financial support as follows (principal investigator). These sources had no role in the preparation of data or the manuscript. No funding was obtained from the tobacco industry.Federal funding via Region Stockholm(A.L.: ALF\#20140309) and Västra Götaland Region (A.L.: LUA \#141851); King Gustaf V's and Queen Victoria's Freemason Research Fund (A.L.); Swedish Heart-Lung Foundation (A.L.: \#20180315); Swedish Research Council (A.L.: \#2016-01563); Swedish Society for Respiratory Medicine (A.L.); Swedish Heart-Lung Foundation (M.S.: \#20160367); Swedish Heart-Lung Foundation (J.B.: \#20150365); and the King Gustaf V's 80-year foundation (J.B.: \#FAI-2015-0165).

\section{Author Contributions}

M.S., K.C., I.Q., J.B., and A.L. outlined the clinical and experimental study concepts. The study subjects were identified, recruited, and examined by A.A., H.A., and I.Q. The clinical samples were collected and investigated by M.S., K.C., and L.B. as well as by M.P. under the supervision by S.L. M.S. performed the main bulk of neutrophil surface marker analyses, with support from K.C., and summarized all datasets. All authors reviewed the completed datasets. The manuscript was outlined, completed, and revised by M.S., J.B., and A.L. All authors critically reviewed, commented, and approved the manuscript prior to its submission.

\section{References}

1 Mathers CD, Loncar D. Projections of global mortality and burden of disease from 2002 2030. PLoS Med. 2006;3(11):e442.

2 GOLD. Global strategy for the diagnosis, management and prevention of COPD, global initiative for chronic obstructive lung disease (GOLD). 2017 : https://goldcopd.org.

3 Barnes PJ. New anti-inflammatory targets for chronic obstructive pulmonary disease. Nat Rev Drug Discov. 2013;12(7):543-59.

4 Droemann D, Goldmann T, Tiedje T, Zabel P, Dalhoff K, Schaaf B. Toll-like receptor 2 expression is decreased on alveolar macrophages in cigarette smokers and COPD patients. Respir Res. 2005;6(1):68.

5 Zhang Y, Geng S, Prasad GL, Li L. Suppression of neutrophil antimicrobial functions by total particulate matter from cigarette smoke. Front Immunol. 2018;9:2274.
6 Herr C, Beisswenger C, Hess C, Kandler K, Suttorp N, Welte T, et al. Suppression of pulmonary innate host defence in smokers. Thorax. 2009;64(2):144-9.

7 Andelid K, Andersson A, Yoshihara S, Åhrén C, Jirholt P, Ekberg-Jansson A, et al. Systemic signs of neutrophil mobilization during clinically stable periods and during exacerbations in smokers with obstructive pulmonary disease. Int J Chron Obstruct Pulmon Dis. 2015;10:1253-63.

8 Stanescu D, Stănescu A, Veriter C, Kostianev S, Calcagni PG, Fabbri LM, et al. Airways obstruction, chronic expectoration, and rapid decline of FEV1 in smokers are associated with increased levels of sputum neutrophils. Thorax. 1996;51(3):267-71.

9 Pesci A, Majori M, Cuomo A, Borciani N, Bertacco S, Cacciani G, et al. Neutrophils infiltrating bronchial epithelium in chronic ob- structive pulmonary disease. Respir Med. 1998;92(6):863-70.

10 Stockley RA. Neutrophils and the pathogenesis of COPD. Chest. 2002;121(5 Suppl):151S-5S.

11 Hoenderdos K, Condliffe A. The neutrophil in chronic obstructive pulmonary disease. Am J Respir Cell Mol Biol. 2013;48(5):531-9.

12 Agustí A. Systemic effects of chronic obstructive pulmonary disease: what we know and what we don't know (but should). Proc Am Thorac Soc. 2007;4(7):522-5.

13 Zarember KA, Kuhns DB. Editorial: will the real neutrophil please stand up? J Leukoc Biol. 2011;90(6):1039-41.

14 Sengeløv H, Kjeldsen L, Diamond MS, Springer TA, Borregaard N. Subcellular localization and dynamics of Mac-1 (alpha m beta 2) in human neutrophils. J Clin Invest. 1993. 92(3):1467-76. 
15 Kuhns DB, Long Priel DA, Gallin JI. Loss of L-selectin (CD62L) on human neutrophils following exudation in vivo. Cell Immunol. 1995;164(2):306-10.

16 Davidsson L, Björkman L, Christenson K, Alsterholm M, Movitz C, Thorén FB, et al. A simple skin blister technique for the study of in vivo transmigration of human leukocytes. J Immunol Methods. 2013;393(1-2): 8-17.

17 Padra M, Andersson A, Levänen B, Premaratne $\mathrm{P}$, Asgeirsdottir $\mathrm{H}$, Tengvall S, et al. Increased MUC1 plus a larger quantity and complex size for MUC5AC in the peripheral airway lumen of long-term tobacco smokers. Clin Sci. 2020;134(10): 1107-1125. https://pubmed.ncbi.nlm.nih. gov/32400877/.

18 Walter RE, Wilk JB, Larson MG, Vasan RS, Keaney JF, Lipinska I, et al. Systemic inflammation and COPD: the Framingham Heart Study. Chest. 2008;133(1):19-25.

19 Lokwani R, Wark PA, Baines KJ, Fricker M, Barker D, Simpson JL. Blood neutrophils in COPD but not asthma exhibit a primed phenotype with downregulated CD62L expression. Int J Chron Obstruct Pulmon Dis. 2019; 14:2517-25.
20 Blidberg K, Palmberg L, James A, Billing B, Henriksson E, Lantz AS, et al. Adhesion molecules in subjects with COPD and healthy non-smokers: a cross sectional parallel group study. Respir Res. 2013;14(1):47.

21 Björkman L, Christenson K, Davidsson L, Mårtensson J, Amirbeagi $\mathrm{F}$, Welin $\mathrm{A}$, et al. Neutrophil recruitment to inflamed joints can occur without cellular priming. J Leukoc Biol. 2019;105(6):1123-30.

22 Condliffe AM, Chilvers ER, Haslett C, Dransfield I. Priming differentially regulates neutrophil adhesion molecule expression/function. Immunology. 1996;89(1):105-11. 